\title{
DISINTEGRATION OF MEASURES ON COMPACT TRANSFORMATION GROUPS
}

\author{
BY RUSSELL A. JOHNSON
}

Communicated by Alexandra Bellow, June 1, 1976

The present work falls into two parts. In the first, a left transformation group [2] $(G, X)$ with $G$ a compact metric group and $X$ a locally compact Hausdorff space is given; in the second, a bitransformation group [2] $(G, X, T)$ with $G, X$ compact Hausdorff and $T$ arbitrary is considered. It is always assumed that $G$ acts freely; thus $g \cdot x=x$ implies $g=$ identity in $G(x \in X)$.

1. Let $\pi: X \rightarrow X / G \equiv Y$ be the projection. Let $\mu$ be a Radon measure on $X, \nu=\pi(\mu)$.

1.1. TheOREM. There is a disintegration [1], $\lambda: y \rightarrow \lambda_{y}$ of $\mu$ with respect to $\pi$ such that

(a) $\lambda_{y}$ is supported on $\pi^{-1}(y)$;

(b) $\lambda$ is $\nu$-Lusin-measurable

(thus, if $K \subset Y$ is compact, there is a countable collection $K_{i}$ of compact sets, with $\nu\left(K \sim \bigcup_{i=1}^{\infty} K_{i}\right)=0$, such that $\lambda \mid K_{i}$ is continuous for each $\left.i\right)$. If $\lambda^{\prime}$ is another disintegration of $\mu$ with respect to $\pi$ satisfying (a) and (b), then $\lambda^{\prime}=\lambda$ v-a.e.

To prove 1.1, one first assumes $X$ is compact and $G$ is a Lie group. In this case, $X$ is "measure-theoretically" the product $Y \times G$; this follows from the existence of local cross-sections to the projection $\pi$ [6] . Let $\pi_{2}: X \cong Y \times G$ $\rightarrow G$, and define a map $\xi$ from $L^{1}(Y, \nu)$ to the space of Radon measures on $G$ as follows: $\xi(f)=\pi_{2}[(f \circ \pi) \cdot \mu]$. Apply the Dunford-Pettis Theorem [3] to $\xi$ to obtain a map $\omega$ from $Y$ to $M_{+}(G)=$ the set of positive Radon measures $\eta$ on $G$ such that $\|\eta\|=1$. The map $\lambda$ is easily obtained from $\omega$. One now completes the proof by (i) approximating $G$ by a sequence of Lie groups [6]; (ii) using the fact that there is a locally countable collection of pairwise disjoint compact subsets of $Y$ the complement of whose union is locally $\nu$-null [1].

2. First suppose $G$ is metric. Let $\mu$ be a T-ergodic measure on $X$, and let $\lambda$ be a disintegration of $\mu$ as in 1.1. Let $G \supset G_{0}=\left\{g \in G \mid \int_{X} f(g x) d \mu(x)=\right.$ $\int_{X} f(x) d \mu(x)$ for all $\left.f \in C(X)\right\} ; G_{0}$ is a closed subgroup of $G$. Denote the normalized Haar measure on $G_{0}$ by $\gamma_{0}$.

AMS (MOS) subject classifications (1970). Primary 28A50, 28A65, 54H 20. 
2.1. Theorem. For each $y \in Y$, there exists $x \in \pi^{-1}(y)$ such that $\int_{X} f d \lambda_{y}$ $=\int_{G} f(g x) d \gamma_{0}(g)(f \in C(X))$.

Thus each $\lambda_{y}$ "looks like" $\gamma_{0}$.

To prove 2.1, define $\phi_{x}: G \rightarrow X: g \rightarrow g \cdot x$ for each $x \in X$. Then $\phi_{x}$ is a homeomorphism onto $\pi^{-1} \pi(x)$. Define $F: X \rightarrow M_{+}(G): F(x)=\phi_{x}^{-1}\left(\lambda_{y}\right)$ where $y=\pi(x)$.

2.2. Lemma. Let $H$ map $X$ to a Hausdorff space, and suppose (i) $H$ is $\mu$ Lusin-measurable, (ii) $H(x t)=H(x) \mu$-a.e. for each fixed $t \in T$. Then $H=$ const $\mu$-a.e.

This lemma may be applied to $F$; thus $F(x)=$ const $\mu$-a.e. Results of [5] now imply that $F(x)=\gamma_{0} \mu$-a.e., and 2.1 follows immediately.

If $G$ is not metric, our results are quite a bit weaker. We do have an analogue of 2.1, however, if $Y$ has a strong lifting [3]. Let $M_{0}(X)=\{\eta: \eta$ is a positive Radon measure on $X,\|\eta\|=1, \eta$ is $G_{0}$-invariant . It is easily seen that 2.1 is equivalent with the following

2.3. Statement. For each $y \in Y, \lambda_{y}$ is extreme in the compact convex set $M_{0}(X)$.

2.4. ThEOREM. Suppose Y has a strong lifting [3]. Then there exists a weakly measurable disintegration $\lambda$ such that (i) $\lambda_{y}$ is supported on $\pi^{-1}(y)$; (ii) $\lambda_{y}$ is extreme in $M_{0}(X)$ for all $y$.

The proof is a straightforward argument using 2.1 and approximation of $G$ by Lie groups.

These results represent a portion of the author's doctoral thesis [4]. He wishes to thank his advisor, Professor Robert Ellis, for his many valuable suggestions and his constant encouragement.

\section{REFERENCES}

1. N. Bourbaki, Éléments de mathématique, Integration, Fasc. XIII, XXI, XXV, $2 \mathrm{nd}$. ed., Livre VI, Chaps. 1-4, 5, 6, Actualités Sci. Indust., nos. 1175, 1244, 1281, Hermann, Paris, 1965, 1956, 1959. MR 36 \#2763; 18, 881; 23 \#A2033. \#2463.

2. R. Ellis, Lectures on topological dynamics, Benjamin, New York, 1969. MR 42

3. A. Ionescu-Tulcea and C. Ionescu-Tulcea, Topics in the theory of lifting, SpringerVerlag, Berlin and New York, 1969. MR 43 \#2185.

4. R. Johnson, Topological and measure-theoretic properties of compact transformation groups with free action, Thesis, Univ. of Minnesota, 1975.

5. H. B. Keynes and $O$. Newton, The structure of ergodic measures for compact group extensions, Israel J. Math. (to appear).

6. D. Montgomery and L. Zippin, Topological transformation groups, Interscience, New York, 1955. MR 17, 383.

DEPARTMENT OF MATHEMATICS, UNIVERSITY OF MSCONSIN-PARKSIDE, KENOSHA, WISCONSIN 53140 